\title{
A Workflow for Extracting Plot-level Biophysical Indicators From Aerially Acquired Multispectral Imagery
}

Matthew Wallhead and Heping Zhu, U.S. Department of Agriculture-Agriculture Research Service, Application Technology Research Unit, Wooster, OH 44691; John Sulik, MicaSense, Inc., Seattle, WA 98103; and William Stump, Department of Plant Sciences, University of Wyoming, Laramie 82071

Accepted for publication 18 April 2017.

The use of unmanned aerial vehicles (UAVs) for precision agriculture and research has been increasing in recent years. Advances in technologies associated with UAVs have allowed researchers, farmers, and agribusinesses to incorporate UAVs coupled with various imaging systems into data-collection activities and aid in the decision-making process. Multispectral imagery allows for a quantitative assessment of biophysical indicators or plant health status. High spatial and temporal resolutions allow for a vast amount of data to be gathered on-demand. Data generated from multispectral, aerialacquired imagery can complement ground-based measures of plant health status or crop performance (i.e., disease severity, incidence, vigor, stand count, stress, and yield).

Remote-sensing techniques for landscape-level imagery have been well-established (Hatfield and Pinter 1993; Jackson 1984; Rock et al. 1986; Rouse et al. 1974). What is needed now is a standardized workflow for the quantitative assessment of plant biophysical indicators using low-altitude, high-resolution, UAV-acquired, multispectral imagery. With this need in mind, the authors developed and proposed a workflow (Fig. 1) for extracting plot-level vegetationindex means utilizing orthomosaics generated from a multirotor UAV coupled with a scientific-grade multispectral camera.

\section{Flight Planning, Image Acquisition, and Processing}

Images should be acquired at an altitude as near to ground level as feasible while ensuring avoidance of obstructions. Due to the arrangement of the individual sensors within a discrete-band multispectral camera, there is usually a minimum flying height above the crop canopy that must be observed to ensure that image sets can be successfully processed into an orthomosaic. Spatial resolution (pixels/ $\mathrm{cm}$ ) is influenced greatly by the flying height above the plant canopy. Additionally, it is necessary to program flight plans with enough overlap and sidelap between images to ensure that the photogrammetry software finds enough tie points to successfully mosaic an image set. A tie point is a point that represents the same location in the overlap between adjacent images and is used to link images to create orthomosaics.

\section{Extraction of Plot-level Data}

Vegetation indices (VIs) are plant biophysical indicators that are combinations of two or more bands and reduce the dimensionality

Corresponding author: Matthew Wallhead; E-mail: wallhead.3@gmail.com

*The $\boldsymbol{e}$-Xtra logo stands for "electronic extra" and indicates that one supplementary figure is published online.

This article is in the public domain and not copyrightable. It may be freely reprinted with customary crediting of the source. The American Phytopathological Society, 2017. of the dataset. VI values can be added to datasets containing groundbased measurements. To extract data at the plot level, first an orthomosaic is loaded as a raster layer in QGIS V2.18 (QGIS Development Team). Then a shapefile is created as a vector layer where the user will delimit the area of interest or plots by adding features to the orthomosaic (Fig. 2). Next, a customized $\mathrm{R}$ script (Supplemental Table S1) is used in R V3.3.2 ( $\mathrm{R}$ Core Team) to calculate zonal statistics and extract VIs at the plot level. The user sets the working directory and is prompted by the $\mathrm{R}$ script to select the shapefile defining the plots and orthomosaic being used. One consideration the user must make that can affect the VI values considerably is whether to choose to mask, i.e., exclude certain pixel values. Pixel values that

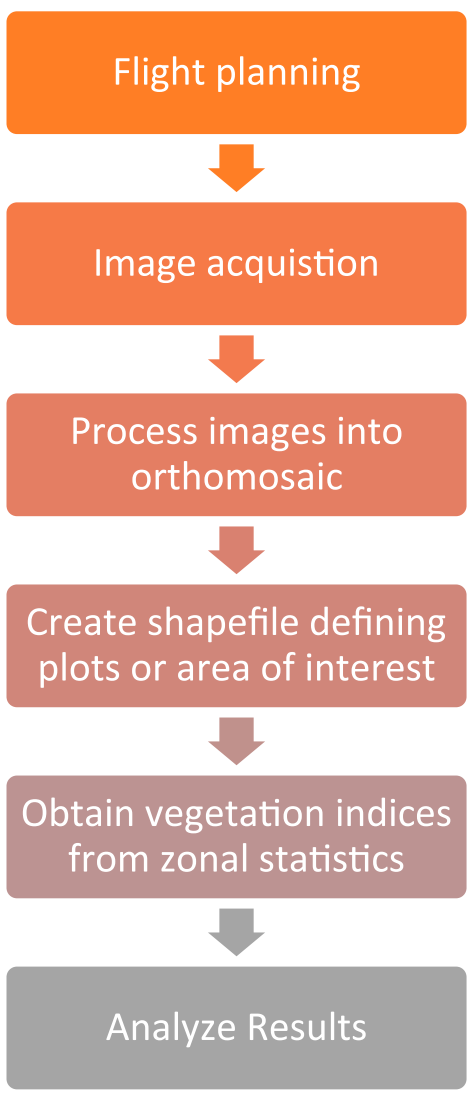

FIGURE 1

A workflow for extracting plot-level biophysical indicators from aerial imagery. 


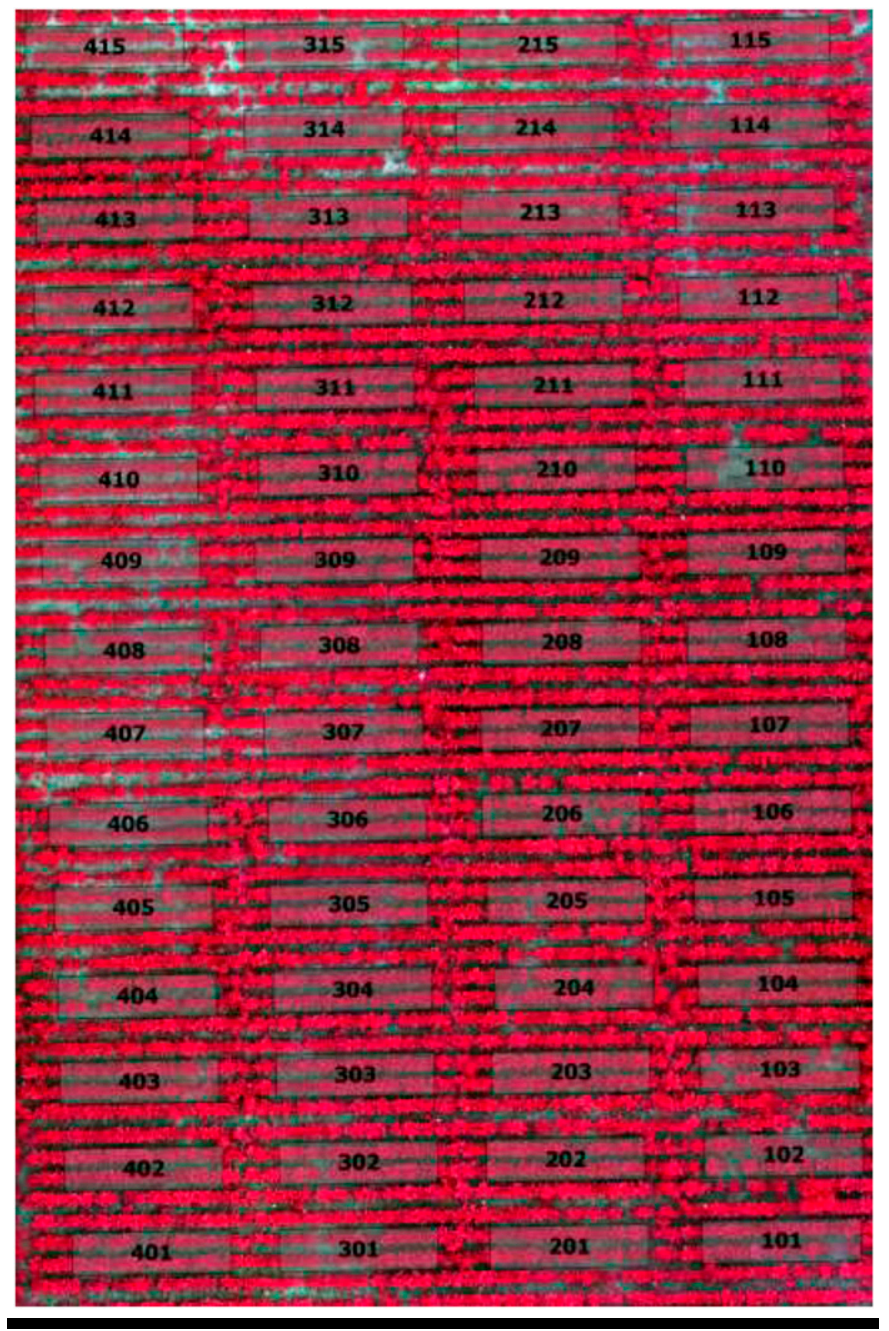

FIGURE 2

Example of a color-infrared orthomosaic with a shapefile defining experimental plots overlaid.

a user may choose to mask out of the dataset includes pixel values corresponding to soil, man-made materials, and shade. Pixel values in the orthomosaic can be determined by using the Value Tool available in QGIS. The output from the script includes average values for the individual band and the mean VI values for each plot as a text file. At this point, the user can import the text file into Microsoft Excel as a comma-separated (.csv) file and the data can be analyzed accordingly.

\section{Criteria for Inclusion in Analysis}

Individuals utilizing VIs obtained from orthomosaics are encouraged to be prudent regarding the inclusion of the data into larger datasets. They are also encouraged to only use data with high significance values $(P<0.0001)$ and consider the Bayes factor and posterior probabilities as outlined in Madden et al. (2015). Pairwise comparisons of least-square means can then be completed by using Tukey's honestly significant differences (HSD) test using the GLIMMIX procedure in SAS 9.4 (SAS Institute Inc., Cary, NC).

\section{Summary and Implications}

Judicious use of multispectral imagery and vegetation indices gives researchers, farmers, and agribusinesses additional metrics by which to gauge plant health status and aid expert systems for decision-making. In addition, UAVs can aid in targeted application of farm inputs and support variable-rate technologies. Targeted applications and use of variable-rate technologies can assist in closing yield gaps or managing pesticide application schedules by accessing disease, insect, and weed pressures. Yield gaps are differences between observed yields and what is attainable for a given region. As the use of UAVs and associated data collection activities expands, it will become increasingly important that data are being properly incorporated and utilized by ag professionals.

\section{Literature Cited}

Hatfield, P. L., and Pinter, P. J. 1993. Remote sensing for crop protection. Crop Prot. 12:403-413

Jackson, R. D. 1984. Remote sensing of vegetation characteristics for farm management. SPIE Proc. 475:81-96.

Madden, L. V., Shah, D. A., and Esker, P. D. 2015. Does the $P$ value have a future in plant pathology? Phytopathology 105:1400-1407.

Rock, B. N., Vogelmann, J. E., Williams, D. L., Vogelmann, A. F., and Hoshizaki, T. 1986. Remote detection of forest damage. Bioscience 36: 439-445.

Rouse, J., Jr., Haas, R. H., Schell, J. A., and Deering, D. W. 1974. Monitoring vegetation systems in the Great Plains with ERTS. NASA Spec. Publ. 351: 309-317. 